\title{
The LUCID-Timepix Spacecraft Payload and the CERN@school Educational Programme
}

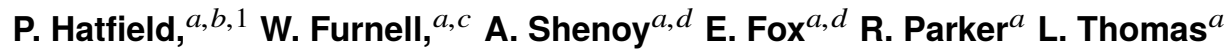 \\ ${ }^{a}$ The Institute for Research in Schools \\ Wellcome Wolfson Building, 165 Queen's Gate, London, SW7 5HD, UK \\ ${ }^{b}$ Clarendon Laboratory, University of Oxford \\ Parks Road, Oxford OX1 3PU, UK \\ ${ }^{c}$ School of Computing, University of Kent \\ Canterbury, Kent, CT2 7NF, UK \\ ${ }^{d}$ The Langton Star Centre \\ Langton Lane, Nackington Road, Canterbury, Kent, CT4 7AS, UK \\ E-mail: peter.hatfield@physics.ox.ac.uk
}

\begin{abstract}
The Langton Ultimate Cosmic ray Intensity Detector (LUCID) is a payload onboard the satellite TechDemoSat-1, used to study the radiation environment in Low Earth Orbit ( $\sim 635 \mathrm{~km})$. LUCID operated from 2014 to 2017, collecting over 2.1 million frames of radiation data from its five Timepix detectors on board. LUCID is one of the first uses of the Timepix detector technology in open space, with the data providing useful insight into the performance of this technology in new environments. The data has been analysed using computing resources provided by GridPP, with a novel machine learning algorithm. For managing the LUCID data, we have developed an online platform called Timepix Analysis Platform at School (TAPAS). This provides a swift and simple way for users to analyse data that they collect using Timepix detectors from both LUCID and other school based Timepix projects. These projects constitute a secondary school programme 'CERN@school' that give a framework for novel implementations of conventional classroom experiments using Timepix, as well as letting students contribute to large international scientific collaborations and devise their own research projects.
\end{abstract}

Keywords: Space instrumentation; Charged particle detection; Radiation monitoring; Analysis and statistical methods

\footnotetext{
${ }^{1}$ Corresponding author.
} 


\section{Contents}

1 Introduction 1

2 LUCID 2

2.1 The Payload 2

2.1.1 TechDemoSat-1 2

2.1.2 Instrument Design 2

2.2 Analysis 3

2.2.1 The TAPAS Data Analysis and Visualisation Tool 3

2.2.2 Metric Based Network 3

2.3 Results 4

3 CERN@school and Educational Programme 6

4 Conclusions 6

\section{Introduction}

The late 1990s saw the advent of photon counting pixel detectors for radiation detection with the development of the Medipix detectors [1,2]. Recently, there has been increased interest in their application in space $[3,4,5,6]$. In particular their ability to distinguish between different particle types and give angular information have, for example, proved valuable in understanding the radiation environment of the ISS. Seven NASA/IEAP-developed Radiation Environment Monitors (REMs), Timepix detectors in compact USB mounting, have been deployed to the ISS (altitude $\sim 400 \mathrm{~km}$ ) [7, $3,8]$. Four of these have been in near continuous operation since 2012, operated via an onboard laptop. The second use of Medipix in space was on the European Space Agency (ESA) Proba V mission, launched on the 7th May 2013 to Low Earth Orbit (LEO) with an altitude of 820km, with the spacecraft payload Space Application of Timepix Radiation Monitor (SATRAM, [4]) onboard. SATRAM carries a single Timepix detector and is operating and continuously taking data today. In addition, Exploration Flight Test 1 (EFT-1), the first flight of the Orion Multi-Purpose Crew Vehicle (MPCV) on a two orbit, 4.5 hour trip on the 5th December 2014 took Medipix data the farthest from Earth to date at $\sim 5910 \mathrm{~km}$ [5]. Most recently, on the 23rd June 2017 the cubesat VZLUSAT-1 (altitude 510km, [9,6]) carried a miniaturised x-ray telescope, that uses Timepix detectors [10], into orbit for astrophysical, space weather studies, and terrestrial X-ray monitoring applications [11]. Future planned missions include: a particle telescope architecture containing two Timepix detectors in sync, on the Rapid International Scientific Experiment Satellite (RISESAT, $[12,13])$, a Japanese FIRST mission to orbit at $\sim 700 \mathrm{~km}$, further Medipix being sent to the ISS, HERA monitors (units containing single Timepix detectors being developed at NASA for use on 
future MPCV missions), the proposed Biosentinel astrobiology deep-space cubesat mission and on trans-lunar NASA-ORION missions in the 2020s.

In these proceedings we report the first results from one of the early uses of Medipix in orbit (and the first on a commercial platform, and the first with Medipix detectors in a 3D configuration), the Langton Ultimate Cosmic ray Intensity Detector (LUCID, [14]) on board TechDemoSat-1. In addition, LUCID is linked to a extensive programme of education and research in the classroom; we describe this programme, known as CERN@ school [15], and associated data dissemination tools.

\section{LUCID}

\subsection{The Payload}

\subsubsection{TechDemoSat-1}

LUCID is a payload on the technology demonstration satellite TechDemoSat-1 (TDS-1, see figure 1). The project started in 2008, and was developed as a collaboration between Langton Star Centre secondary school student researchers, the Medipix Collaboration, and Surrey Satellite Technology Limited (SSTL), who built both LUCID and TDS-1. Much of the subsequent operations, data management and analysis were led by secondary school researchers through the Institute for Research in Schools (IRIS), with support from SSTL and the Medipix collaboration ${ }^{1}$. LUCID is part of the TDS-1 Space Environment Suite, which consists of the Miniature Radiation Environment and effects Monitor (MuREM, [16, 17]), the Charged Particle Spectrometer (ChaPS, [18]) and the Highly Miniaturized Radiation Monitor (HMRM, [19, 20]). TDS-1 launched on 8 July 2014 (15:58:28 UTC) on a Soyuz-2-1b launch vehicle with Fregat-M upper stage from the Baikonur Cosmodrome in Kazakhstan, into a $635 \mathrm{~km}, 98.4^{\circ}$ Sun-synchronous orbit.

LUCID began data collection shortly after launch, and data collection ceased on the 4th July 2017. TDS-1 operations have now ended, and at some point in the medium-term it will be deorbited by the Icarus-1 Cranfield Drag Augmentation System de-orbiter [21] which will over the next 25 years guide the spacecraft into the Earth's atmosphere, where it will disintegrate.

The detectors used in LUCID are based on the Timepix ASIC chip [22, 23], part of the second generation of Medipix (Medipix2, [24, 22, 25]). The detectors used are equipped with a $300 \mu \mathrm{m}$ silicon sensor. The Timepix chips contain $256 \times 256$ pixels, each measuring $55 \mu \mathrm{m}$ on each side, giving a total collecting area of $1.98 \mathrm{~cm}^{2}$. LUCID was always run in ToT mode.

\subsubsection{Instrument Design}

We briefly summarise the instrument design here; more detailed design information of LUCID can be found in [14] and the LUCID System Design Document (D. Cooke, SSTL, private communication). The payload has five Timepix radiation detectors in a cube-like configuration (see Fig. 1b), with four detectors orthogonally positioned facing outwards (TPX0 through TPX3), and the fifth in the centre (TPX4), facing outwards (relative to the centre of LUCID). A photograph of the instrument is shown in Figure 1. The chips were surrounded by a $0.75 \mathrm{~mm}$ thick aluminium dome which blocks intense light, plasma and low-energy charged particles. LUCID is mounted on the 'Earthside' of TDS-1.

\footnotetext{
${ }^{1}$ Alongside generous support from many other individuals and organisations, see Acknowledgements.
} 
a)

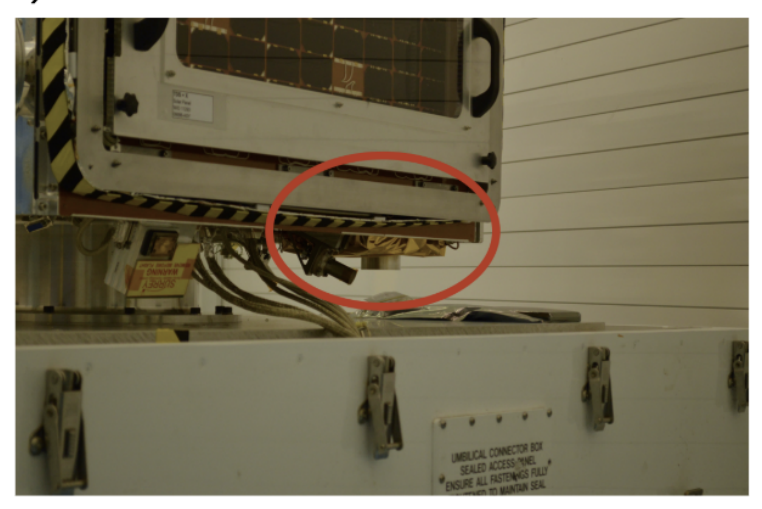

b)

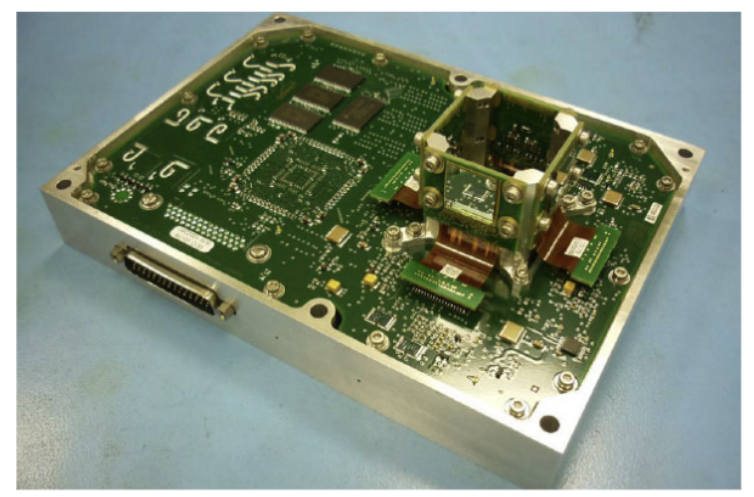

Figure 1. LUCID shown integrated on TDS-1 in the SSTL assembly room b) the LUCID payload showing the Timepix detector array (taken from the LUCID System Design Document). The four orthogonally positioned detectors are visible on the right hand side of the instrument.

The detectors were calibrated by the Institute of Experimental and Applied Physics (IEAP) at the Czech Technical University in Prague. The calibration process involves exposing the detectors to $\mathrm{X}$ rays of discrete energy, and modelling the low energy end non-linear response of each individual pixel $[26,27]$. The performance and expected measurements of LUCID were simulated in GEANT4 $[28,29]$.

\subsection{Analysis}

\subsubsection{The TAPAS Data Analysis and Visualisation Tool}

We have developed The Timepix Analysis Platform at School (TAPAS) to allow secondary school student researchers across the UK to analyse and share the data that they gather using Timepix radiation detectors across all CERN@ @school projects, and additionally as a home for the particle count data from the LUCID experiment. The platform allows users to upload their own data taken with a Timepix detectors, or data which has been provided to them, such as the TimPix ISS-REM radiation data. Students can conduct further analysis using their own choice of software packages or even by choosing to write their own, in a programming language they are most comfortable with using. All of the LUCID particle count data is downloadable as CSV files from TAPAS.

\subsubsection{Metric Based Network}

To analyse the data, first a clustering algorithm is run on the LUCID frames to identify individual particle tracks. Then a Metric Based Network (MBN) approach to classifying the particles was used; a small number of easily computed features were calculated for each track, and the particles were classified based on these metrics using a multi-layer neural network [30]. The hyper-parameters (number of hidden layers, number of nodes in each hidden layer etc.) for this architecture were optimised manually.

To generate training data for classifying particle tracks, a web application called LUCID Trainer was created. It allows volunteer classifiers (typically IRIS secondary school researchers) to simply 


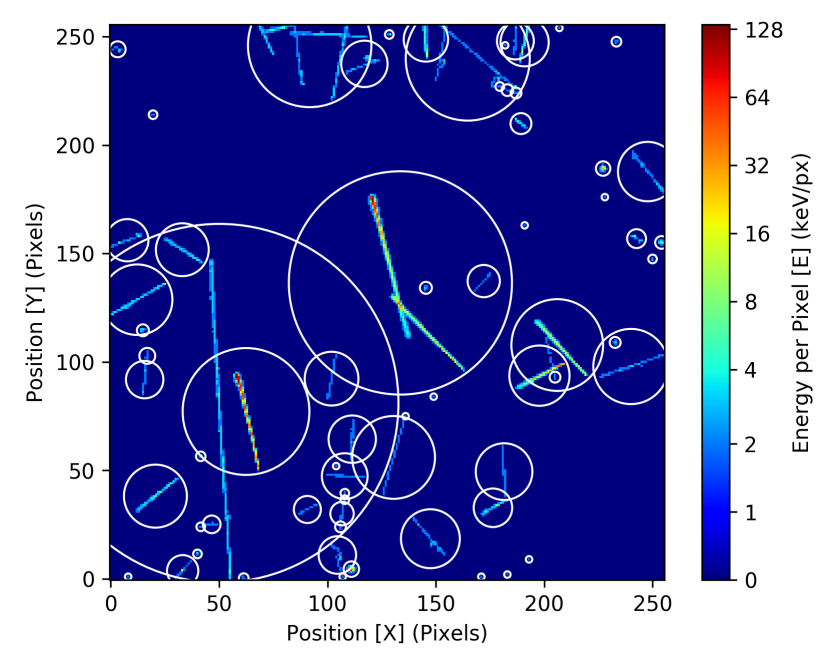

Figure 2. An example LUCID frame with individual particle tracks identified.

click through automatically generated questionnaires. The resulting training set is thus based on human classifications, which as a methodology is necessarily less accurate than using a training set constructed from known calibrated sources. Therefore quoted particle counts and fluxes for LUCID calculated in this way necessarily have some caveats. Nevertheless, these preliminary initial results still give us a good overview of the distribution of morphology of detections, and in the future calibrated classifications can be generated and used for training to obtain more realistic particle counts [31].

Figure 3 shows the confusion matrices for our algorithm, the Metric-Based Network, and an Analytic Classifier (where tracks are classified based on an analytic function of the feature metrics, used in an early stage of the analysis of the data [32]) to show how machine learning leads to much better performance. The Metric-Based Network algorithm performs well, with the only substantial misclassifications being 30-40\% of muons and protons being misclassified as electrons - partially because of similarities in the track shapes, partially because electrons dominate the overall sample, and partially because the labels used in the training are imperfect.

\subsection{Results}

We show in figure 4 the number of particles detected over the Earth's surface for $~ 4000$ frames from 2016-08-17 to 2016-09-21 for electrons (although similar figures were generated for other particles). Higher radiation levels (by more than a factor of ten) around South Atlantic Anomaly (an area of known increased radiation flux, centred at roughly $30^{\circ} \mathrm{S}, 60^{\circ} \mathrm{W}$ ) and the poles (roughly $60^{\circ} \mathrm{N}, 60^{\circ} \mathrm{S}$ ) are clearly evident for all particle types.

We present some early scientific results (e.g. a comparison of measurements 'dayside' and 'nightside') and analysis in [14] illustrating that the experiment appears to give reasonable results as a prelude to future work investigating particle isotropy and the physics of particle transport from the sun and the trapped electron model. 

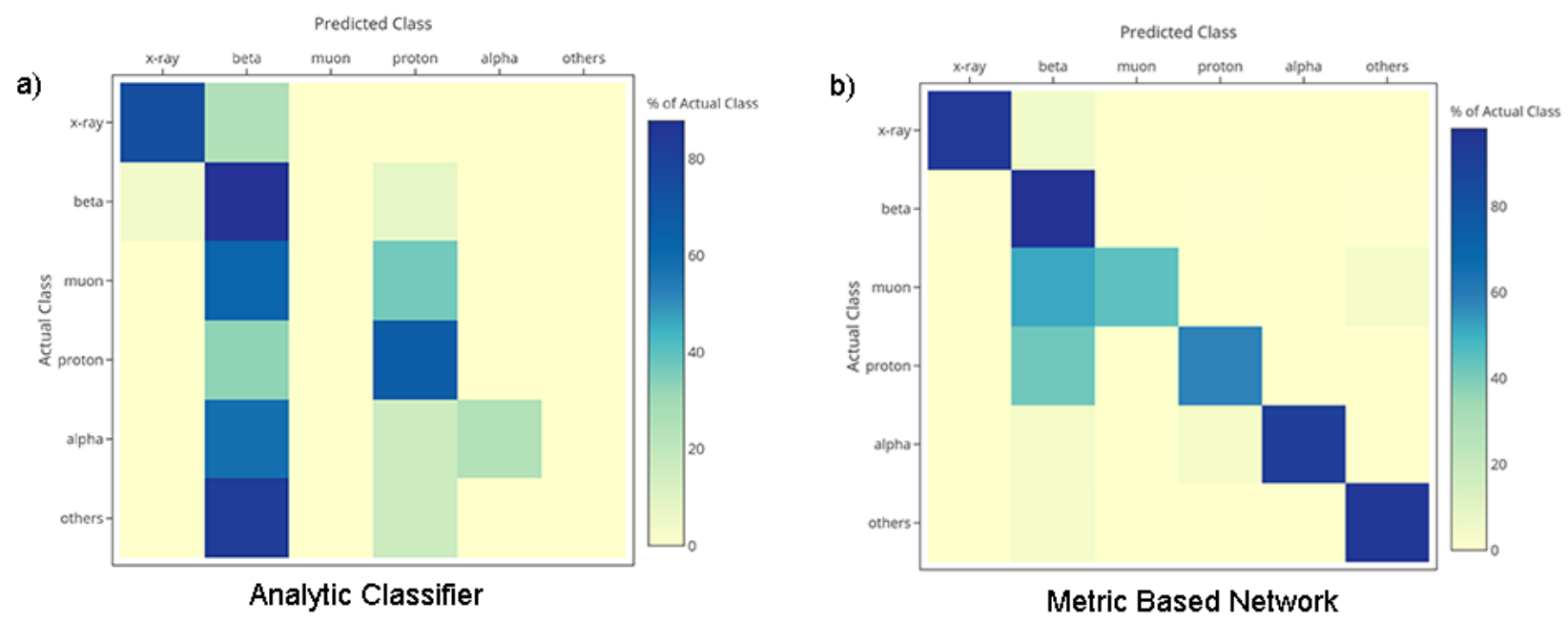

Figure 3. Confusion matrices for the analytic classifier and MBN. Squares in the grid are colour coded by percentage of 'actual class' classified as 'predicted class'.

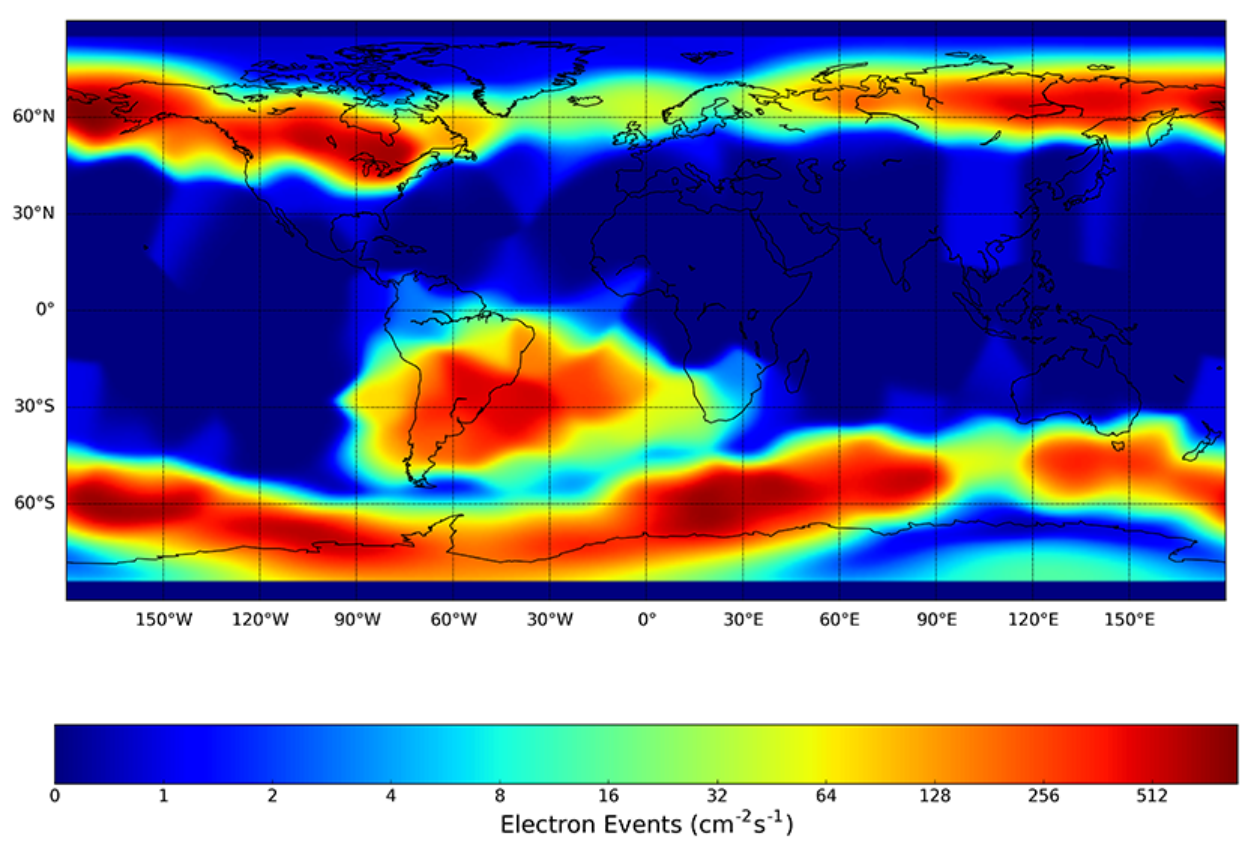

Figure 4. Radiation map for electrons, 2016-08-17 to 2016-09-21. 


\section{CERN@school and Educational Programme}

Alongside the role of UK secondary schools in LUCID, Institute for Research in Schools (IRIS) school student researchers ${ }^{2}$ have also worked analysing ISS REM data under the guise of 'TimPix'. TimPix was part of Mission Principia, a larger scheme of school and educational projects linked to British ESA Astronaut Tim Peake's trip to the ISS 15th December 2015 to 18th June 2016 as part of Expeditions 46 and 47.

LUCID and TimPix are part of a larger multi-disciplinary suite of projects and experiments using Medipix technology being tested and applied in schools (see also RasPIX). The CERN@ school project is a framework for secondary school pupils in the UK to use Medipix. This has been used for both novel tests of traditional classroom experiments (e.g. inverse square law [33]) as well as original science, for example the Radiation In Soil Experiment (RISE) which has measured the radiation in different geological samples across the UK, and construction of a robotic threedimensional radiation scanner [34]. CERN@ school kits (Timepix detector, laptop and resources) are lent out for six week periods at a time and are used by more than a hundred schools every year.

Other ongoing applications within this framework include applying data reduction techniques developed for LUCID and Timpix to data from Medipix detectors in large particle physics experiments. For example student researchers have been involved with efforts to detect magnetic monopoles in the MoEDAL experiment [35] and observe the two-photon Breit-Wheeler process ("turning light into matter") for the first time at the Central Laser Facility [36], both using Timepix. Finally CERN@ sea is a project that has deployed Medipix RasPix detectors on a wave propelled Unmanned Surface Vessel, 'AutoNaut'.

Collectively these projects show the ability for school based scientific projects to successfully develop new applications and techniques for new technology in a range of environments, alongside playing a key role in education $[37,38,39]$. All of these projects are managed through the same data storage and reduction pipeline TAPAS, see section 2.2.1.

CERN@school can be viewed as an important sociological test of the use of Medipix technology. Medipix had previously been predominantly used by professional scientists. CERN@school was the first widespread use of Medipix across 100s of institutions, with users ranging from novices to experts. The challenges of handling data from a very heterogeneous set of sources for use by a wide variety of users of different levels of expertise is a test bed for any future hypothetical largescale use of Medipix outside of academia and research labs e.g. Medipix as a personal radiation monitor in a nuclear power plant or for nuclear medicine workers.

\section{Conclusions}

In these proceedings we introduce the LUCID detector (the third use of Medipix detectors in space, the second in open space, and the first on a commercial platform and with a 3D configuration) that flew aboard TDS-1 taking data from 2014 to 2017. We describe the data pipeline from data collection to reduced catalogue of classified particles, a novel machine learning particle track classification algorithm, and some early science results. We also discuss LUCIDs links to the larger CERN@school educational ecosystem.

\footnotetext{
2Predominantly aged $11-18$ years old.
} 


\section{Acknowledgments}

The authors and IRIS are extremely grateful to the Medipix Collaboration and SSTL for ten years of support, in particular we are hugely thankful to Dr Michael Campbell (CERN/Medipix Collaboration), Prof. Larry Pinksy (University of Houston/NASA), David Cooke (SSTL), Dr Stuart Eves (SSTL) and Dr Jonathan Eastwood (Imperial). Specific thanks to Prof. Stanislav Pospisil and IEAP CTU Prague for their support, in particular for calibrating the Timepix detectors used in LUCID.

Alongside the authors, the following students have also contributed substantially over the history of the project: Toby Freeland, Matt Harrison, Cal Hewitt, Sam Kittle, Nick Liu, Rachel O’Leary, Rachel Powell, Adam Sandey, Hector Stalker, Tom Stevenson and Cassie Warren, as well as extensive support and encouragement over many years from Dr Tom Whyntie (Oxford). Thank you also to students who contributed to the classifications used in the training set for the machine learning classifications, and the many other students who worked on LUCID over the last ten years.

AS and EF would like to thank Mr Rupert Champion (Langton Star Centre) and Dr Tim Lesworth (Langton Star Centre) for providing ongoing support with their IRIS activities. PH acknowledges funding from the Engineering and Physical Sciences Research Council.

The authors would like to thank Prof. Steven Rose (Imperial/Oxford) and Dr Carlos Granja (Advacam, Prague) for their helpful comments, remarks and input to the project. The authors are also grateful to Steven Greenwood (IRIS) who is a key part of LUCID, CERN@ @school and IRIS reaching so many students.

The authors and the Institute for Research in Schools would like to acknowledge generous support from: Humphrey Battcock, The Science and Technology Facilities Council, The Science Museum, The Institute of Physics, The Royal Commission for the Exhibition of 1851, The Ogden Trust, CERN, The Medipix Collaboration, NASA, the UK Space Agency, Kent County Council, IEAP CTU Prague, SEEDA and the GridPP Collaboration (in particular Dr Dan Traynor and Queen Mary University of London for use of their GPU and storage resources, and Professor Steve Lloyd for supporting and enabling schools to work with GridPP).

\section{References}

[1] M. G. Bisogni, M. Campbell, M. Conti, P. Delogu, M. E. Fantacci, E. H. M. Heijne et al., Performance of a 4096-pixel photon counting chip, vol. 3445, pp. 298-304, International Society for Optics and Photonics, 11, 1998. DOI.

[2] M. Campbell, E. Heijne, G. Meddeler, E. Pernigotti and W. Snoeys, A readout chip for a $64 x 64$ pixel matrix with 15-bit single photon counting, IEEE Transactions on Nuclear Science $\mathbf{4 5}(6,1998)$ 751-753.

[3] M. Kroupa, A. Bahadori, T. Campbell-Ricketts, A. Empl, S. M. Hoang, J. Idarraga-Munoz et al., A semiconductor radiation imaging pixel detector for space radiation dosimetry, Life Sciences in Space Research 6 (7, 2015) 69-78.

[4] C. Granja, S. Polansky, Z. Vykydal, S. Pospisil, A. Owens, Z. Kozacek et al., The SATRAM Timepix spacecraft payload in open space on board the Proba-V satellite for wide range radiation monitoring in LEO orbit, Planetary and Space Science 125 (6, 2016) 114-129.

[5] R. Gaza, M. Kroupa, R. Rios, N. Stoffle, E. R. Benton and E. J. Semones, Comparison of novel active semiconductor pixel detectorwith passive radiation detectors during the NASA Orion Exploration Flight Test 1 (EFT-1), Radiation Measurements 106 (11, 2017) 290-297.

[6] M. Urban, O. Nentvich, V. Stehlikova, T. Baca, V. Daniel and R. Hudec, VZLUSAT-1: Nanosatellite with miniature lobster eye $X$-ray telescope and qualification of the radiation shielding composite for space application, Acta Astronautica 140 (11, 2017) 96-104.

[7] D. Turecek, L. Pinsky, J. Jakubek, Z. Vykydal, N. Stoffle and S. Pospisil, Small Dosimeter based on Timepix device for International Space Station, Journal of Instrumentation 6 (12, 2011) C12037-C12037. 
[8] N. Stoffle, L. Pinsky, M. Kroupa, S. Hoang, J. Idarraga, C. Amberboy et al., Timepix-based radiation environment monitor measurements aboard the International Space Station, Nuclear Instruments and Methods in Physics Research Section A: Accelerators, Spectrometers, Detectors and Associated Equipment 782 (5, 2015) 143-148.

[9] V. Dániel, L. Pína, A. Inneman, V. Zadražil, T. Báča, M. Platkevič et al., Terrestrial gamma-ray flashes monitor demonstrator on CubeSat, vol. 9978, p. 99780D, International Society for Optics and Photonics, 9, 2016. DOI.

[10] T. Baca, M. Platkevic, J. Jakubek, A. Inneman, V. Stehlikova, M. Urban et al., Miniaturized X-ray telescope for VZLUSAT-1 nanosatellite with Timepix detector, Journal of Instrumentation 11 (10, 2016) C10007-C10007.

[11] L. Pina, R. Hudec, A. Inneman, D. Cerna, J. Jakubek, L. Sieger et al., X-ray monitoring for astrophysical applications on Cubesat, vol. 9510, p. 951005, International Society for Optics and Photonics, 5, 2015. DOI.

[12] T. Kuwahara, K. Yoshida, Y. Sakamoto, Y. Tomioka and K. Fukuda, Satellite system integration based on Space Plug and Play Avionics, in 2011 IEEE/SICE International Symposium on System Integration (SII), pp. 896-901, IEEE, 12, 2011. DOI.

[13] C. Granja, M. Platkevic, D. Turecek, V. Kraus, S. Pospisil, J. Jakubek et al., Timepix-Based Miniaturized Radiation Micro-Tracker for the Micro-Satellite RISESAT, Transactions of the Japan Society for Aeronautical and Space Sciences, Aerospace Technology Japan 12 (2014) Tr_7-Tr_11.

[14] W. Furnell, A. Shenoy, E. Fox and P. Hatfield, First results from the LUCID-Timepix spacecraft payload onboard the TechDemoSat-1 satellite in LEO, submitted, Advances in Space Research (2018) .

[15] R. Parker, Education with Timepix, in prep. (2018).

[16] B. Taylor, C. Underwood, A. Dyer, C. Ashton, S. Rason and J. Browning, The Micro Radiation Environment Monitor (MuREM) and SSTL Radiation Monitor (SSTL RM) on TechDemoSat-1, IEEE Transactions on Nuclear Science 59 (8, 2012) 1060-1065.

[17] C. Underwood, B. Taylor, A. Dyer, A. Hands, K. Ryden, W. Avison et al., Development of miniaturised radiation environment monitors for commercial spacefight: Flight results from the MuREM/RM payloads, in 2016 16th European Conference on Radiation and Its Effects on Components and Systems (RADECS), pp. 1-8, IEEE, 9, 2016. DOI.

[18] D. O. Kataria, A. Fazakerley, L. Green, S. Mathews, H. Hu, M. Hailey et al., In-situ sensors and instrumentation for Space Weather and the Sunjammer Solar Sail mission opportunity, .

[19] E. F. Mitchell, H. M. Araújo, E. Daly, N. Guerrini, S. Gunes-Lasnet, D. Griffin et al., The Highly Miniaturised Radiation Monitor, Journal of Instrumentation 9 (7, 2014) P07010-P07010.

[20] N. Guerrini, R. Turchetta, D. Griffin, T. Morse, A. Morse, O. Poyntz-Wright et al., Design and characterisation of a highly miniaturised radiation monitor HMRM, Nuclear Instruments and Methods in Physics Research Section A: Accelerators, Spectrometers, Detectors and Associated Equipment 731 $(12,2013)$ 154-159.

[21] S. Hobbs, J. Kingston, P. Roberts, C. Juanes, R. Sewell, B. Snapir et al., De-Orbit Sail Design for TechDemoSat-1. 1, 2013.

[22] X. Llopart, R. Ballabriga, M. Campbell, L. Tlustos and W. Wong, Timepix, a 65k programmable pixel readout chip for arrival time, energy and/or photon counting measurements, Nuclear Instruments and Methods in Physics Research Section A: Accelerators, Spectrometers, Detectors and Associated Equipment 581 (10, 2007) 485-494. 
[23] R. Plackett, T. Tick, R. Ballabriga, S. Vahanen, D. Turecek, M. Campbell et al., Current status of the Medipix2, Timepix, Medipix3 and Timepix2 pixel readout chips, PoS VERTEX2010 (2010) 030.

[24] X. Llopart, M. Campbell, R. Dinapoli, D. San Segundo and E. Pernigotti, Medipix2: A 64-k pixel readout chip with 55- $\mu$ m square elements working in single photon counting mode, IEEE Transactions on Nuclear Science 49 (10, 2002) 2279-2283.

[25] R. Ballabriga, G. Blaj, M. Campbell, M. Fiederle, D. Greiffenberg, E. H. M. Heijne et al., Characterization of the Medipix3 pixel readout chip, Journal of Instrumentation $6(1,2011)$ C01052-C01052.

[26] J. Jakubek, A. Cejnarova, T. Holy, S. Pospisil, J. Uher and Z. Vykydal, Pixel detectors for imaging with heavy charged particles, Nuclear Instruments and Methods in Physics Research Section A: Accelerators, Spectrometers, Detectors and Associated Equipment 591 (6, 2008) 155-158.

[27] J. Jakubek, Precise energy calibration of pixel detector working in time-over-threshold mode, Nuclear Instruments and Methods in Physics Research Section A: Accelerators, Spectrometers, Detectors and Associated Equipment 633 (5, 2011) S262-S266.

[28] T. Whyntie and M. A. Harrison, Simulation and analysis of the LUCID experiment in the Low Earth Orbit radiation environment, Journal of Physics: Conference Series 513 (6, 2014) 022038.

[29] T. Whyntie and M. Harrison, Full simulation of the LUCID experiment in the Low Earth Orbit radiation environment, Journal of Instrumentation 10 (3, 2015) C03043-C03043.

[30] S. Haykin, Neural Networks: A Comprehensive Foundation. Prentice Hall PTR, Upper Saddle River, NJ, USA, 2nd ed., 1998.

[31] C. Granja, J. Jakubek, S. Polansky, V. Zach, P. Krist, D. Chvatil et al., Resolving power of pixel detector Timepix for wide-range electron, proton and ion detection, Nuclear Instruments and Methods in Physics Research Section A: Accelerators, Spectrometers, Detectors and Associated Equipment (2018) .

[32] T. Whyntie, H. Bithray, J. Cook, A. Coupe, D. Eddy, R. Fickling et al., CERN@ school: demonstrating physics with the Timepix detector, Contemporary Physics 56 (10, 2015) 451-467.

[33] T. Whyntie and B. Parker, Investigating the inverse square law with the Timepix hybrid silicon pixel detector: a CERN@school demonstration experiment, Physics Education 48 (5, 2013) 344-349.

[34] T. Whyntie, J. Cook, A. Coupe, R. L. Fickling, B. Parker and N. Shearer, CERN@ school: Bringing CERN into the classroom, Nuclear and Particle Physics Proceedings (2016) .

[35] J. L. Pinfold, Searching for the magnetic monopole and other highly ionizing particles at accelerators using nuclear track detectors, Radiation Measurements 44 (10, 2009) 834-839.

[36] O. J. Pike, F. Mackenroth, E. G. Hill and S. J. Rose, A photon-photon collider in a vacuum hohlraum, Nature Photonics (2014) .

[37] D. Colthurst, M. Kent, R. Mauchaza, T. Senasinghe, G. Sparkes, F. Vasilijevic et al., Research science in schools: An insight into the student experience in biomedical science and particle physics, School Science Review 97 (2015) 4451.

[38] B. Parker, Real science, real classrooms, School Science Review (2017) .

[39] B. Parker, E. Fox and L. Rushton, IRIS extending young peoples participation and attainment in STEM and promoting teachers and students as vital members of STEM research communities, 2018. 